\title{
USO DE UN COMPLEJO ENZIMÁTICO Y UN BIOPROTECTOR COMERCIAL EN LA ESTABILIDAD Y TRANSFORMACIÓN DE EXCRETAS PORCINAS
}

\author{
USE OF ENZYME COMPLEX AND COMMERCIAL BIOPROTECTOR IN STABILITY AND \\ TRANSFORMATION OF PIG MANURE
}

\author{
${ }^{1}$ Carmen Alvarez S., ${ }^{2}$ Juan Juscamaita M. y ${ }^{3}$ Jeannett Noa L.
}

\begin{abstract}
Resumen
El presente trabajo de investigación se realizó teniendo como objetivos: Estabilizar y transformar las excretas porcinas, en biofertilizante: biol (fracción líquida) y biosol (fracción sólida), mediante el tratamiento con un complejo enzimático y un bioprotector comercial, medido a través de análisis microbiológico, fisicoquímico y pH. Se realizó en dos etapas: en la primera, para hidrolizar las excretas, se trabajaron tres porcentajes de enzimas $(0 \%, 0.1 \%, 0.5 \%$ y $0.7 \%)$ a $65^{\circ} \mathrm{C}$, eligiéndose el nivel de $0.5 \%$ por ser con el que obtuvo la mayor cantidad de sólidos solubles totales (SST) expresados en grados Brix; en la segunda etapa los tratamientos consistieron en la combinación de diferentes niveles $(10 \%, 15 \%$ y $20 \%$ ) de un bioprotector comercial (MO) y melaza (M) completando al 100\% con las excretas hidrolizadas (E), teniendo así 9 combinaciones más un tratamiento control. Para el análisis del índice de pH se utilizó el Diseño Completo al Azar (DCA) y para la comparación de medias se utilizó la prueba de Tukey. Los tratamientos fueron evaluados por 30 días, observándose en ellos su estabilidad, $\mathrm{pH}$, color y olor, para seleccionar los mejores tratamientos, los cuales fueron T4 (15B 10M 75E), T7 (20B 10M 70E), T8 (20B 15M 65E) y T9 (20B 20M 60E), quienes al quinto día presentaron valores de $\mathrm{pH}$ de 4.3, 3.97, 3.94 y 3.98 respectivamente. Al realizar el recuento de bacterias coliformes, Salmonella y Staphylococcus de una muestra combinada de los 4 tratamientos seleccionados se encontró un valor de $<1 \times 10^{1} \mathrm{UFC} / \mathrm{ml}$ para los tres tipos de bacterias; en el análisis fisicoquímico se halló valores de $4.24 \% \mathrm{~N}, 5.01 \%$ P2O5 y $1.08 \% \mathrm{~K}_{2} \mathrm{O}$ para el biosol y 5320 $\mathrm{mg} / \mathrm{L}$ de $\mathrm{N}$ total, $2964 \mathrm{mg} / \mathrm{L}$ de $\mathrm{P}$ total y $8850 \mathrm{mg} / \mathrm{L}$ de $\mathrm{K}$ total para el biol. El uso de un complejo enzimático sobre las excretas porcinas favorece la acción del bioprotector comercial acelerando así el proceso de fermentación homoláctica. El pH de las excretas tratadas con complejo enzimático y complejo de bacterias acidolácticas sufre una disminución a niveles menores a 4.5, estabilizándose al primer día de tratamiento. El tratamiento T4 (15B 10M 75E), resultó ser el mejor tratamiento, debido a que presentó un notorio descenso de $\mathrm{pH}$, cercano a 4 al quinto día de fermentación, y se mostró estable, además de ser el tratamiento más rentable económicamente. El análisis microbiológico de los productos obtenidos muestra que no existe microorganismos patógenos en ellos.
\end{abstract}

Palabras claves: Excretas porcinas, complejo enzimático, bacterias ácido lácticas.

\section{Summary}

This research was conducted with the following objectives: to stabilize and transform pig manure in biofertlizer: biol (liquid fraction) and biosol (solid fraction) treated with an enzymatic complex and commercial bioprotector, measured through microbiological, physicochemical and $\mathrm{pH}$ analysis. It was performed in two stages: first, to hydrolyzed excreta, three percentages of enzyme $(0 \%, 0.1 \%, 0.5 \%$ and $0.7 \%)$ at $65^{\circ} \mathrm{C}$ were used, the level of $0.5 \%$ was chosen because it had the highest amount of total soluble solids (TSS) expressed in brix degrees; in the second stage treatments they consisted of the combination of different levels $(10 \%, 15 \%$ and $20 \%)$ of commercial bioprotector (MO) and molasses (M) completing $100 \%$ with hydrolyzed excret, thus having nine combinations plus a control treatment. For the analysis of $\mathrm{pH}$ index was used the Complete Random Design (DCA) and Tukey test comparison was used. The treatments were evaluated for 30 days, showing them their stability, $\mathrm{pH}$, color and smell, to select the best treatment, which were T4 (15B 10M 75E), T7 (20B 10M 70E), T8 (20B 15M 65E) and T9 (20B 20M 60E), the fifth day who had pH values of 4.3, 3.97, 3.94 and 3.98, respectively. The count of coliforme bacteria, Salmonella and Staphylococcus of a combined simple of the 4 treatments selected showed value of $<1 \times 10^{1} \mathrm{CFU} / \mathrm{ml}$ for the three types of bacteria; in physico-chemical analysis values of $4.24 \%$ $\mathrm{N}$ was found, $5.01 \% \mathrm{P}_{2} \mathrm{O}_{5}$ and $1.08 \% \mathrm{~K}_{2} \mathrm{O}$ for biosol and $5320 \mathrm{mg} / \mathrm{L}$ of total N, $2964 \mathrm{mg} / \mathrm{L}$ total P and $8850 \mathrm{mg} / \mathrm{L}$ total K for biol. Using an enzyme complex on swine manure favors of commercial bioprotector action accelerating homolactic fermentation process. The $\mathrm{pH}$ of the manure treated with enzyme complex and of acid lactic bacterial complex suffers a decline to levels below 4.5, stabilizing the first day of treatment. The T4 (15B 10M 75E), treatment turned out to be the best treatment, because it showed a marked decrease in ph, close to 4 , on the fifth day of fermentation, and was stable, besides being the most economically profitable treatment. Microbiological analysis of the products obtained shows no pathogenic microorganisms in them.

Key word: swine manure, enzyme complex, acid lactic bacterial.

\footnotetext{
${ }^{1}$ Profesora Asociada- Dpto. Producción Animal-Facultad de Zootecnia, Universidad Nacional Agraria La Molina, Lima, Perú. E-mail: chas@ lamolina.edu.pe.

${ }^{2}$ Profesor Asociado-Dpto. Biología-Facultad de Ciencias, Universidad Nacional Agraria La Molina, Lima, Perú. E-mail: jjm@lamolina.edu.pe. ${ }^{3}$ Ing. Zootecnista-Práctica Privada
} 


\section{Introduccion}

Una de las perspectivas de la porcicultura nacional es el aumento de la demanda actual de la carne de cerdo, razón por la cual muchas de las granjas tecnificadas tienden a incrementar su producción; pero se ve limitada, en parte, por problemas en el manejo de los residuos, tanto sólidos como líquidos (Peralta, 2010; Chia- Fang, 2009). Si bien es cierto hay muchas alternativas que le dan valor agregado a la gestión de manejo de residuos, no todas ellas pueden ser utilizadas fácilmente por el alto costo de su instalación, implementación y mantenimiento (COCIL, 2007).

La gestión de control de residuos sólidos se basa mayormente en el manejo de las excretas, debido a que es la fuente de mayor contaminación del agua, aire y suelo, causando un grave impacto ambiental. La contaminación del aire es el mayor problema generado por las granjas porcinas debido a la proximidad de ellas a los centros urbanos, poniendo así en riesgo su permanencia en la zona, a causa de las denuncias de los pobladores por los olores, insectos y roedores; problema que ha llevado a que muchas granjas tengan que cambiar de ubicación hacia un lugar más alejado de la urbe (Schiffman et al, 2001; Zahn et al., 2001; Cornejo, 2011).

Por lo tanto en la actualidad se busca nuevas alternativas para el manejo de las excretas, de bajo costo y fácil acceso, que den un mayor valor agregado teniendo de esta manera una mejor retribución económica y una de estas alternativas es el uso de Lactobacillus en el tratamiento de excretas, habiéndose empleado para tratar excretas de ganado lechero y efluentes porcinos (Peralta, 2010; Cornejo, 2011). La obtención de biofertilizantes: Biol y Biosol es una de las alternativas. El biol, también denominado bio abono líquido, es un tipo de abono orgánico que es producto de la fermentación anaeróbica de materiales orgánicos provenientes de animales y vegetales, como estiércol o restos vegetales (Buchelli, 2014). El biosol, es el resultado de separar la parte sólida del "Fango" resultante de la fermentación anaeróbica dentro del Fermentador o Biodigestor. Dependiendo de la tecnología a emplear, este subproducto tratado puede tener entre $25 \%$ y $10 \%$ de humedad. Su composición depende mucho de los residuos que se emplearon para su fabricación (Aparcana, 2008).

El objetivo del presente trabajo fue estabilizar y transformar las excretas porcinas en biofertilizante: biol (fracción líquida) y biosol (fracción sólida), mediante el tratamiento con un complejo enzimático y un bioprotector comercial, medido a través de análisis microbiológico, fisicoquímico y $\mathrm{pH}$.

\section{Materiales y métodos}

Lugar de ejecución. El trabajo experimental se realizó en dos etapas, ambas se realizaron en el Laboratorio de Biorremediación del Departamento de Biología de la Facultad de Ciencias de la Universidad Nacional Agraria La Molina, distrito de La Molina, provincia de Lima, departamento de Lima.
Materia prima e insumos. La materia prima e insumos utilizados fueron: excretas porcinas proveniente de los corrales de las áreas de reproducción, maternidad y engorde de la Unidad Experimental en Cerdos (UEC) de la Universidad Nacional Agraria La Molina (UNALM); complejo enzimático comercial constituido básicamente por enzimas proteolíticas de origen bacteriano, específicamente endopeptidasa (subtilisina) y el complejo de bacterias ácido láctico.

Tratamientos y Metodología Experimental. En la primera etapa se empleó una muestra de $100 \mathrm{~g}$ de excreta porcina a la que se le agregó enzima (proteasa microbiana), en tres niveles $(0.1 \% ; 0.5 \%$ y $0.7 \%)$, con la finalidad de determinar el porcentaje más apropiado para obtener el mayor nivel de sólidos solubles totales (SST) en grados Brix ( ${ }^{\circ}$ Brix). Se evaluó durante cuatro horas la variación de SST, para obtener la excreta hidrolizada (E) que será empleada en la siguiente etapa. En la segunda etapa los tratamientos consistieron en la combinación de diferentes niveles $10 \%, 15 \%$ y $20 \%$ de bioprotector comercial (MO) y melaza (M) completando al $100 \%$ con las excretas hidrolizadas (E), teniendo así 9 combinaciones más un tratamiento control, siendo éstos: T1 (10MO 10M $80 \mathrm{E}), \mathrm{T} 2$ (10MO 15M 75E), T3 (10MO 20M 70E), T4 (15MO 10M 75E), T5 (15MO 15M 70E), T6 (15MO 20M 65E), T7 (20MO 10M 70E), T8 (20MO 15M 65E), T9 (20MO 20M 60E), T10 (0MO 0M 100E), los cuales fueron evaluados por 30 días, observándose en ellos su estabilidad, $\mathrm{pH}$, color y olor. Luego se seleccionaron los mejores tratamientos y a los productos obtenidos de ellos, biol y biosol, se les realizó análisis microbiológico, fisicoquímico y proximal.

Análisis estadístico. Para comparar los resultados de $\mathrm{pH}$ de los tratamientos se empleó un Diseño Completo al Azar (DCA) con diez tratamientos y tres repeticiones y para determinar diferencias significativas entre los tratamientos, se empleó la Prueba de Comparación de Medias de Tukey.

\section{Resultados y discusión}

\section{Primera etapa:}

Obtención de Excretas Hidrolizadas. A partir de la primera hora de evaluación se observa que no existen diferencias estadísticas $(\mathrm{p}<0.05)$ entre los niveles de enzima $0.5 \%$ y $0.7 \%$, pero si entre éstos y $0.1 \%$ y control (Tabla 1). Ante estos resultados se seleccionó el nivel de $0.5 \%$ ya que es económicamente más rentable, al tener los mismos resultados que el nivel $0.7 \%$, pero usando una menor cantidad de enzima. 
Tabla 1. Variación de los sólidos solubles totales expresados en grados Brix, en cuatro horas de evaluación, de acuerdo al porcentaje de inclusión de un complejo enzimático.

\begin{tabular}{lllllll}
\hline $\begin{array}{r}\text { Nivel de } \\
\text { enzima, } \%\end{array}$ & $0^{\circ}$ hora & $1^{\circ}$ hora & $2^{\circ}$ hora & $3^{\circ}$ hora & $4^{\circ}$ hora \\
\hline T0 & 0.0 & $4.00^{\mathrm{a}}$ & $4.00^{\mathrm{c}}$ & $4.17^{\mathrm{c}}$ & $4.33^{\mathrm{c}}$ & $4.83^{\mathrm{c}}$ \\
T1 & 0.1 & $4.00^{\mathrm{a}}$ & $5.17^{\mathrm{b}}$ & $5.33^{\mathrm{b}}$ & $6.00^{\mathrm{b}}$ & $6.17^{\mathrm{b}}$ \\
T2 & 0.5 & $4.00^{\mathrm{a}}$ & $6.17^{\mathrm{a}}$ & $6.33^{\mathrm{a}}$ & $7.17^{\mathrm{a}}$ & $7.83^{\mathrm{a}}$ \\
T3 & 0.7 & $4.00^{\mathrm{a}}$ & $6.17^{\mathrm{a}}$ & $6.50^{\mathrm{a}}$ & $7.33^{\mathrm{a}}$ & $8.00^{\mathrm{a}}$ \\
\hline
\end{tabular}

${ }^{\text {abc }}$ Letras iguales dentro de una misma columna indican que no hay diferencias significativas entre tratamientos a la Prueba de Tukey.

Fuente: Elaboración propia

\section{Segunda etapa:}

Evaluación del parámetro de $\mathrm{pH}$ en los tratamientos. En la Tabla 2 se presentan los valores de $\mathrm{pH}$ de los tratamientos, desde el día 0 hasta el día 30, pudiéndose observar que en el día 0 existen diferencias significativas $(\mathrm{p}<0.05)$ entre los tratamientos a excepción de los tratamientos T2, T4 у T8; T3 у T6; T1 у T6; T1 у T5 quienes son estadísticamente similares entre sí. Todos los tratamientos comienzan con medidas de $\mathrm{pH}$ por encima de 5.4, el mayor valor de $\mathrm{pH}$ corresponde al tratamiento T10, que es el tratamiento control, y a medida que se va incorporando los porcentajes de melaza y complejo de bacterias ácido lácticas, correspondientes a cada tratamiento, los valores de $\mathrm{pH}$ van variando hacia un $\mathrm{pH}$ más ácido, estas diferencias se deben al pH de los insumos empleados y a su nivel de inclusión en cada tratamiento. Al día 1, se observa un descenso del $\mathrm{pH}$ en casi todos los tratamientos excepto el tratamiento T10, éste al contrario de los demás tratamientos registró un aumento de $\mathrm{pH}$, debido al proceso de descomposición que ocurre sobre la materia orgánica presente. El tratamiento T7 fue el que registró el mayor descenso de $\mathrm{pH}$ al día 1, a pesar de haber sido el tratamiento que comenzó con el nivel más alto de $\mathrm{pH}$ después del control.

Al día 5 se observa diferencias estadísticas significativas $(\mathrm{p}<0.05)$ entre los valores de $\mathrm{pH}$ de los tratamientos $\mathrm{T} 4$, T7, T8 y T9 con respecto a los demás tratamientos, siendo estos tratamientos los que obtuvieron los valores más bajos de $\mathrm{pH}$ (4.03, 3.97, 3.94 y 3.98, respectivamente). Estos tratamientos además de presentar los valores de $\mathrm{pH}$ más bajos para el día 5 son los que presentaron también un mayor descenso de los valores de $\mathrm{pH}$. Estos resultados indican que el valor inicial de $\mathrm{pH}$ no afecta su posterior descenso, sólo es necesario darles las condiciones necesarias para que las bacterias ácido lácticas puedan iniciar el proceso de fermentación homoláctica, es decir, proporcionarles un medio anaerobio y un sustrato adecuado para que puedan colonizar el medio (Betancourt et al., 2005 citado por Cornejo, 2011). Del día 5 al 30 se evaluó la estabilidad del pH de todos los tratamientos, observándose mínimas variaciones en todos los tratamientos a excepción del control.

Tabla 2. Valores de $\mathrm{pH}$ de los tratamientos obtenidos entre el día 0 y el día 30 de evaluación.

\begin{tabular}{|c|c|c|c|c|c|c|c|c|c|}
\hline & \multirow{2}{*}{ Tratamiento } & \multicolumn{8}{|c|}{ Medidas de $\mathrm{pH}$} \\
\hline & & Día 0 & Día 1 & Día 5 & Día 10 & Día 15 & Día 20 & Día 25 & Día 30 \\
\hline $\mathrm{T} 1$ & (10B 10M 80E) & $5.47^{\mathrm{gf}}$ & 4.34 & $4.11^{\mathrm{bc}}$ & 4.02 & 4.02 & 4.00 & 4.01 & 3.93 \\
\hline $\mathrm{T} 2$ & (10B 15M 75E) & $5.71^{\mathrm{d}}$ & 4.48 & $4.16^{\mathrm{b}}$ & 4.05 & 4.05 & 4.04 & 3.99 & 3.95 \\
\hline T3 & (10B 20M 70E) & $5.53^{\mathrm{e}}$ & 4.46 & $4.08^{\mathrm{bcd}}$ & 4.01 & 4.00 & 3.99 & 3.92 & 3.71 \\
\hline $\mathrm{T} 4$ & $(15 B$ 10M 75E) & $5.74^{\mathrm{d}}$ & 4.30 & $4.03^{\text {cde }}$ & 3.96 & 3.96 & 4.00 & 3.90 & 3.87 \\
\hline T5 & (15B 15M 70E) & $5.42^{\mathrm{g}}$ & 4.35 & $4.05^{\mathrm{cd}}$ & 4.03 & 3.99 & 3.94 & 3.92 & 3.91 \\
\hline T6 & (15B 20M 65E) & $5.51^{\mathrm{ef}}$ & 4.78 & $4.07^{\mathrm{cd}}$ & 3.99 & 3.96 & 3.88 & 3.88 & 3.84 \\
\hline $\mathrm{T} 7$ & (20B 10M 70E) & $5.93^{b}$ & 4.23 & $3.97^{\mathrm{ed}}$ & 3.96 & 3.91 & 3.84 & 3.83 & 3.79 \\
\hline $\mathrm{T} 8$ & (20B 15M 65E) & $5.71^{\mathrm{d}}$ & 4.28 & $3.94^{\mathrm{e}}$ & 3.90 & 3.88 & 3.80 & 3.80 & 3.78 \\
\hline T9 & (20B 20M 60E) & $5.87^{\mathrm{c}}$ & 4.42 & $3.98^{\text {ed }}$ & 3.99 & 3.90 & 3.80 & 3.81 & 3.77 \\
\hline $\mathrm{T} 10$ & (0B 0M 100E) & $6.55^{\mathrm{a}}$ & 6.67 & $7.53^{\mathrm{a}}$ & 8.20 & 8.43 & 8.73 & 9.19 & 9.26 \\
\hline
\end{tabular}

Fuente: Elaboración propia.

Análisis microbiológico. Como se puede observar en la Tabla 3 las excretas porcinas frescas presentan una alta carga bacteriana inicial (día 0), la cual es conformada en su totalidad por coliformes, siendo el recuento de estos de $22 \times 10^{6} \mathrm{UFC} / \mathrm{ml}$. 
Tabla 3. Análisis microbiológico de las excretas porcinas, biol y biosol obtenidos de los tratamientos T4, T7, T8 y $\mathrm{T} 9$.

\begin{tabular}{|c|c|c|c|}
\hline \multirow[t]{2}{*}{$\begin{array}{c}\text { Análisis } \\
\text { microbiológico }\end{array}$} & \multirow[t]{2}{*}{$\begin{array}{l}\text { Excretas } \\
\text { porcinas } \\
\text { frescas }\end{array}$} & \multicolumn{2}{|c|}{$\begin{array}{c}\text { Productos obtenidos } \\
\text { de las excretas } \\
\text { porcinas con } 5 \text { días de } \\
\text { tratamiento }\end{array}$} \\
\hline & & Biol & Biosol \\
\hline $\begin{array}{l}\text { Recuento de bacterias } \\
\text { coliformes (UFC/ml) }\end{array}$ & $22 \times 10^{6}$ & $<1 \times 10^{1}$ & $<1 \times 10^{1}$ \\
\hline Recuento de bacterias & & & \\
\hline $\begin{array}{l}\text { Salmonella } \quad s p p . \\
\text { (UFC/ml) }\end{array}$ & $<1 \times 10^{1}$ & $<1 \times 10^{1}$ & $<1 \times 10^{1}$ \\
\hline $\begin{array}{l}\text { Recuento de bacterias } \\
\text { St a p hy loc o c c us } \\
\text { aureus.(UFC/ml) }\end{array}$ & $<1 \times 10^{1}$ & $<1 \times 10^{1}$ & $<1 \times 10^{1}$ \\
\hline
\end{tabular}

Los análisis realizados a los productos obtenidos de las excretas con 5 días de tratamiento (biol y biosol) muestran que no hay presencia de coliformes, lo cual se debe a la acción de las bacterias ácido lácticas, quienes debido a su metabolismo homoláctico, producen ácido láctico como único producto de la fermentación ocasionando así una reducción en el $\mathrm{pH}$, lo cual genera un ambiente ácido, poco propicio para la proliferación de bacterias Grampositivas y Gram-negativas (Vázquez et al., 2009). Al no haber bacterias putrefactivas los olores característicos de las excretas disminuyeron en todos los tratamientos (Zahn et al., 2001), evidenciándose esta disminución a partir del primer día y prácticamente para el $5^{\circ}$ día la emanación de olores era casi nula, excepto en el tratamiento T10 (tratamiento control)

Análisis fisicoquímico. En las Tablas 4 y 5 se presenta el análisis fisicoquímico del biol y biosol resultante de los tratamientos seleccionados, observándose que existe una mayor conductividad eléctrica (CE) en el biol con respecto al biosol. La alta CE del biol (27.1 dS/m), puede ser reducida al momento de ser aplicado como biofertilizante, realizando una dilución de $3-5 \mathrm{ml}$ biol /L agua.

El biosol presenta un contenido de $\mathrm{N}, \mathrm{P}_{2} \mathrm{O}_{5}$ y $\mathrm{K}_{2} \mathrm{O}$ de $4.47 \%, 5.01 \%$ y $1.08 \%$, respectivamente y siendo el porcentaje de $\mathrm{K}_{2} \mathrm{O}$ mayor a $1 \%$ y la suma de $\mathrm{N}$ más $\mathrm{P}_{2} \mathrm{O}_{5}$ mayor a 4\%, se puede considerar al biosol como un fertilizante NPK (BOE, 2003).

También se observa que, tanto en el biol como en el biosol, existe presencia de micronutrientes no deseados tales como metales pesados $\mathrm{Cd}, \mathrm{Cr}, \mathrm{Cu}, \mathrm{Pb}, \mathrm{Zn}$, cuyo exceso de uso en el suelo puede generar contaminación por metales pesados y ser perjudicial, por lo cual es necesario un control de la dosis de aplicación para evitar una progresiva acumulación de estos elementos en el suelo.
Análisis proximal. En la Tabla 6 se presenta el análisis proximal de las excretas y biosol, se puede observar que la cantidad de proteína que contiene la excreta porcina es de $17.81 \%$ la cual es menor a lo reportado por Camacho (1998) citado por Padilla (2006) y Gutiérrez y $\mathrm{Ku}(1996)$ quienes reportan valores de $18.75 \%$. Esta variación puede deberse a diferentes factores como a la genética del animal, a la calidad de alimento, la cantidad de proteína digestible presente en el alimento, al tipo de dieta o tipo de formulación, ya que va a variar si se tiene una alimentación por fases y multifases o si se formula en base a proteína ideal (Kerr et al., 2006; den Hartog y Sijtsma, 2007).

La cantidad de proteína presente en el biosol es de 19.7\%, existiendo un incremento en el porcentaje de proteína, con respecto a las excretas, esto debido al metabolismo de las BAL, ya que según ensayos realizados por Mater y Corthier (2004), se observa que las BAL son capaces de iniciar la síntesis de proteína, siempre que tengan condiciones adecuadas.

El contenido de fibra en las excretas y biosol es de 11.46 y $12.68 \%$, valores que son menores a lo reportado por Zanabria (1998) y Rojas (1996) quienes presentan valores de 18.58 y $12.36 \%$ lo cual nos indica que puede ser incluido en la dieta sin reducir la digestibilidad. Los altos contenidos de ceniza se deben al tipo de alimentación que reciben los cerdos, ya que sus dietas son ricas en minerales especialmente $\mathrm{Cu}$ y $\mathrm{Zn}$.

El contenido nutricional del biosol es mucho más rico que el de las excretas porcinas, lo cual nos indica que podemos usarlo en la dieta de los animales y obtener mejores resultados que al incluir las excretas porcinas no tratadas en la dietas, ya que se eliminan algunos factores negativos de las excretas, tales como la presencia de bacterias patógenas y el mal olor característico de ellas que repercute negativamente en la palatabilidad del alimento, pudiéndose mejorar los parámetros reportados por Zanabria (1998) y Rojas (1996) quienes encontraron que los niveles óptimos de inclusión eran $10 \%$ en dietas de cerdos en crecimiento y acabado y niveles de inclusión de $15 \%$ en dietas de engorde de toretes cebuinos, respectivamente.

Análisis costo- beneficio. Se realizó el análisis costo beneficio a cada uno de los tratamientos seleccionados como los mejores tratamientos: T4, T7, T8 y T9. La realización de este análisis se basó en el rendimiento y costo total de cada tratamiento para la producción de biol y biosol y la realización de la proyección de $100 \mathrm{~L}$ de biol, debido a que el biol es el producto que se obtiene en mayor cantidad con respecto al biosol.

Al realizar una análisis de costo - beneficio (Tabla 7) observamos que los tratamientos, T4 y T9, son los que tienen menor costo de producción y por lo tanto son los que generan mayor beneficio, y entre ellos se selecciona al T4 como el tratamiento más rentable. 
Tabla 4. Análisis fisicoquímico de las excretas porcinas y biosol obtenido de los tratamientos T4, T7, T8 y T9 (mezcla compuesta), en base seca.

\begin{tabular}{lcc}
\hline \multirow{2}{*}{ Parámetro } & \multicolumn{2}{c}{ Base seca } \\
\cline { 2 - 3 } & $\begin{array}{c}\text { Excretas } \\
\text { porcinas }\end{array}$ & Biosol cerdo \\
\hline $\mathrm{ms}$ & 100 & 100 \\
$\mathrm{Hd}(\%)$ & 0 & 0 \\
$\mathrm{pH}$ & 6.47 & 4.69 \\
$\mathrm{C} . \mathrm{E}(\mathrm{dS} / \mathrm{m})$ & 8.39 & 16.7 \\
$\mathrm{MO}(\%)$ & 78.38 & 78.66 \\
$\mathrm{~N}(\%)$ & 2.738 & 4.274 \\
$\mathrm{P}{ }_{2} \mathrm{O}_{5}(\%)$ & 4.93 & 5.01 \\
$\mathrm{~K}_{2} \mathrm{O}(\%)$ & 2.07 & 1.08 \\
$\mathrm{CaO}(\%)$ & 6.13 & 5.88 \\
$\mathrm{MgO}(\%)$ & 1.86 & 2.43 \\
$\mathrm{Na}(\%)$ & 0.39 & 0.79 \\
$\mathrm{Fe}(\mathrm{ppm})$ & 574.77 & 3164.52 \\
$\mathrm{Cu}(\mathrm{ppm})$ & 980.25 & 1062.65 \\
$\mathrm{Zn}(\mathrm{ppm})$ & 70.02 & 5161.01 \\
$\mathrm{Mn}(\mathrm{ppm})$ & 380.40 & 339.58 \\
$\mathrm{~B}(\mathrm{ppm})$ & 49.12 & 114.17 \\
$\mathrm{~Pb}$ total $(\mathrm{ppm})$ & 17.64 & 27.90 \\
$\mathrm{Cd}$ total $(\mathrm{ppm})$ & 0.66 & 0.00 \\
$\mathrm{Cr}$ total $(\mathrm{ppm})$ & 9.02 & 15.95 \\
\hline
\end{tabular}

Tabla 5. Análisis físico químico del biol obtenido de los tratamientos T4, T7, T8 y T9 (mezcla compuesta).

\begin{tabular}{lc}
\hline Parámetro & $\begin{array}{c}\text { Biol excretas } \\
\text { porcinas }\end{array}$ \\
\hline $\mathrm{pH}$ & 4.52 \\
$\mathrm{C} . \mathrm{E}(\mathrm{dS} / \mathrm{m})$ & 27.1 \\
Sólidos totales $(\mathrm{g} / \mathrm{L})$ & 210 \\
MO en solución $(\mathrm{g} / \mathrm{L})$ & 161 \\
Macronutrientes & \\
$\mathrm{N}$ total $(\mathrm{mg} / \mathrm{L})$ & 5320 \\
$\mathrm{P}$ total $(\mathrm{mg} / \mathrm{L})$ & 2964 \\
$\mathrm{~K}$ total $(\mathrm{mg} / \mathrm{L})$ & 8850 \\
Ca total $(\mathrm{mg} / \mathrm{L})$ & 6310 \\
Mg total $(\mathrm{mg} / \mathrm{L})$ & 1950 \\
$\mathrm{Na}$ total $(\mathrm{mg} / \mathrm{L})$ & 970 \\
\hline Micronutrientes & \\
\hline Fe total $(\mathrm{mg} / \mathrm{L})$ & 166 \\
Cu total $(\mathrm{mg} / \mathrm{L})$ & 104 \\
$\mathrm{Zn}$ total $(\mathrm{mg} / \mathrm{L})$ & 15.23 \\
Mn total $(\mathrm{mg} / \mathrm{L})$ & 41 \\
$\mathrm{~B}$ total $(\mathrm{mg} / \mathrm{L})$ & 8.47 \\
Cd total $(\mathrm{mg} / \mathrm{L})$ & 0.005 \\
Pb total $(\mathrm{mg} / \mathrm{L})$ & 1.82 \\
Cr total $(\mathrm{mg} / \mathrm{L})$ & 1.055 \\
\hline
\end{tabular}

Fuente: Laboratorio de Análisis de Suelos, Plantas, Aguas y Fertilizantes (LASPAF)
Tabla 6. Análisis proximal de las excretas porcinas y biosol obtenido de los tratamientos T4, T7, T8 y T9 (mezcla compuesta)

\begin{tabular}{lcccc}
\hline \multirow{2}{*}{$\begin{array}{c}\text { Análisis } \\
\text { Proximal }\end{array}$} & \multicolumn{2}{c}{$\begin{array}{c}\text { Base parcialmente } \\
\text { fresca }^{1}\end{array}$} & \multicolumn{2}{c}{ Base seca } \\
\cline { 2 - 5 } & Excretas & Biosol & Excretas & Biosol \\
\hline Humedad \% & 1.58 & 11.03 & - & - \\
Proteína Total & 17.53 & 9.95 & 17.81 & 19.70 \\
(N x 6.25) \% & & 3.67 & 8.07 & 8.92 \\
Grasa \% & 7.94 & 11.42 & 11.46 & 12.68 \\
Fibra Cruda \% & 11.28 & 12.22 & 23.36 \\
Ceniza \% & 20.78 & 11.11 & 21.11 & 23.96 \\
ELN \% & 40.89 & 52.02 & 41.55 & 45.96 \\
\hline
\end{tabular}

Fuente: Laboratorio de Evaluación Nutricional de Alimentos- UNALM ${ }^{1}$ Las muestras fueron pre secadas antes de ser enviadas al laboratorio, por disposición misma del laboratorio.

Tabla 7. Costo-beneficio de los cuatro tratamientos seleccionados para la producción de 100 L de biol.

\begin{tabular}{ccccc}
\hline Tratamiento Producto & Cantidad $\begin{array}{c}\text { Costo } \\
\text { producción, } \\
\text { S/. }\end{array}$ & $\begin{array}{c}\text { Ganancia*, } \\
\text { S/. }\end{array}$ \\
\hline T4 & Biol & 100 L & 163.0 & 287.0 \\
T7 & Biol & 100 L & 188.0 & 262.0 \\
T8 & Biol & 100 L & 194.1 & 255.9 \\
T9 & Biol & 100 L & 163.1 & 271.2 \\
\hline Fuente: Elaboracion propia. & & &
\end{tabular}

*Se considera: Precio venta litro Biol: S/. 4.50

\section{Conclusiones}

El uso de un complejo enzimático sobre las excretas porcinas favorece la acción del bioprotector comercial acelerando así el proceso de fermentación homoláctica. $\mathrm{El} \mathrm{pH}$ de las excretas tratadas con complejo enzimático y bioprotector comercial sufre una disminución a niveles menores a 4.5 , estabilizándose al primer día de tratamiento. El tratamiento T4 (15\% de B-lac, $10 \%$ de Melaza y 75\% de excretas hidrolizadas) resultó ser el mejor tratamiento, debido a que presentó un notorio descenso de $\mathrm{pH}$, cercano a 4 al quinto día de fermentación, y se mostró estable, además de ser el tratamiento más rentable económicamente. El análisis microbiológico de los productos obtenidos muestra que no existe microorganismos patógenos en ellos.

\section{Literatura citada}

Aparcana, S. 2008. Estudio sobre el valor Fertilizante de los Productos del Proceso "Fermentación Anaeróbica" para la Producción de Biogás. German Profec- Perú SAC. Lima. Perú 9p.

Buchelli, H. 2014. Producción de biofertilizante de bagazo de cebada, excretas de vacuno y suero de quesería mediante fermentación homoláctica. Tesis Ing. Ambiental. UNALM. Lima. Perú. 127p. 
Boletín Oficial del Estado Español (BOE), 2003. Real decreto 824/2005 sobre. Productos fertilizantes. Consultado el 21 de enero 2013. Disponible en: http:// www.boe.es/boe/dias/2005/07/19/pdfs/A25592-25669. pdf

Cámara oficial de comercio e industria de Lorca (COCIL), 2007. Guía de Buenas Prácticas Ambientales para el Sector Porcino. Disponible en http://www.1000and100.com/osdc/editor/UserFiles/ File/Guias/GUIA\%20BPA\%20EXPLOTACION\%20 PORCINA.pdf Revisado el 8 de febrero del 2013. 32 p.

Chia- Fang, H. 2009. Toxicidad metanogénica de purín de cerdo mediante ensayos discontinuos. Tesis para optar el título de Ing. Civil Ambiental, Universidad de la Serena, Chile. 97.

Cornejo, M. 2011. Efecto de un Bioprotector Comercial en la Reducción de pH y Carga Microbiana Putrefactiva en Efluentes Porcinos. Tesis Ingeniero Zootecnista Lima, Perú, UNALM. pp. 5-10,19.

Den Hartog y Sijtsma. R. 2007. Estrategias Nutricionales para reducir la contaminación ambiental en la producción de cerdos. XXIII Curso de Especialización FEDNS. Madrid. España. pp.19.45

Gutiérrez, E. y KU, J. 1996. Utilización del Estiércol de Cerdo en la Alimentación de los Rumiantes. Nutrición Animal Tropical 3 (1): 19-41.

Kerr, B.J.; Ziemer, C. J. Trabue, S. L.; Crouse, J. D. and Parkin, T. B. 2006. Manure composition of swine as affected by dietary protein. and cellulose concentrations. J. Anim. Sci. 84:1584-1592

Mater, D. y Corthier, G. 2004. Response of Lactic Acid Bacteria to the Digestive Environment. Journal of Clinical Gastroenterology. 38(2):64-66

Padilla, M. 2006. Algunas consideraciones sobre el manejo y utilización de los remanentes de Granjas Porcinas, Ministerio de Agricultura y Ganadería Dirección De Programas Nacionales Programa Nacional de Cerdos PITTA - Cerdos. Consultado el 22 de enero del 2012. Disponible en: http://www.mag.go.cr/bibliotecavirtual/ prog-cerdos-manejo-remanantes.pdf

Peralta, R. 2010. Determinación de parámetros óptimos en la producción de fast-biol usando las excretas del ganado lechero del establo de la UNALM. Trabajo de investigación. Biólogo. Lima, Perú. UNALM 56-60p.

Rojas, R. 1996. El Uso del Estiércol de Cerdo en el Engorde de Ganado Vacuno. Tesis Ing. Zootecnista. UNALM. Lima, Perú.. 76p.

Schiffman, S. S.; Bennet, J. L. and Raymer, J. H. 2001. Quantification of odors and odorants from swine operations in North Carolina. Agric. For. Meteoro. 108:213-240

Vázquez, S. M., Suárez, H. y Zapata, S., 2009. Utilización de sustancias antimicrobianas producidas por bacterias ácido lácticas en la conservación de la carne. Revista Chilena de Nutrición. 36(1):64-71.
Zahn, J. A., Hatfield, J. L., Laird, D.A., Hart, T. T., Do, Y. S. and Dispirito, A. A. 2001. Functional Classification of Swine Manure Management Systems Based on Effluent and Gas Emission Characteristics. J. Environ. Qual. 30:635-647

Zanabria, C. 1998. Uso de Estiércol Porcino en la Alimentación de Cerdos en Crecimiento y Acabado.Tesis Ing. Zootecnista. Lima, Perú. UNALM.25-27p 